\title{
A jump operator in set recursion
}

Dag Normann

Oslo, April - 77

\section{Introduction.}

The superjump was introduced by Gandy [3] as a type 3 functional that essentially is a uniform jump operator on the type-two functionals. Harrington [5] gave a description of the sets recursive in the superjump ${ }^{3} \mathrm{~S}$. He proved

a Let $\rho^{F}$ be the ordinal for recursion in ${ }^{3} S, 2_{F}$, i.e. $\rho^{F}=w_{1}^{3} S, F$. Then $\rho^{F}$ is the least ordinal recursively Mahlo in $F$ 。

b $I_{\rho}^{F} \cap P(w)=1-S c\left({ }^{3} S,{ }_{F}\right)(=$ those subsets of $w$ recursive in ${ }^{3} S$ and $F$ )

To do this, he defined a notion of strong recursion in the superjump. In his $\mathrm{Ph} . \mathrm{D}$. Thesis this notion is extended to higher type variants of the superjump, ${ }^{\mathrm{k}+3_{\mathrm{S}}}$. Harrington's strong recursion theory in ${ }^{k}+3_{S}, k+2 F$ will have the same total recursive functions, but fewer partial recursive functions. The computation theory will have strong properties such as stage comparison and Grilliot selection.

In Normann [11] we defined a recursion theory on sets called E-recursion. We proved that there are deep connections between E-recursion and kleene-recursion in normal functionals. In this paper we will add a natural scheme of the jump of a relation to the 
schemes of E-recursion. We will call the new theory S-recursion. There will be a similarly deep connection between S-recursion and Harrington's strong recursion in the Superjump, as between E-recursion and Kleene-theory in normal functionals. We will leave this connection unproved, but prove Harrington's results for S-recursion. Some of the arguments are adjustments of ideas from [6], particularly in Theorems 1 and 2 and Iemma 3.

We will use S-recursion to give some characterizations of the envelopes and sections connected to strong recursion in the superjump.

In the sequel we will assume familiarity with set-recursion and the companion theory (theory of codes for sets) for E-recursion. We will concentrate on the special arguments needed for lifting results from E-recursion to S-recursion.

\section{E-recursion and S-recursion}

E-recursion as defined in Normann [11] is obtained by adding indices to the schemes for rudimentary functions, and then a scheme of reflecticn (diagonalization). For a relation $R$ and a set $x$, we defined

$$
\operatorname{Spec}(R ; \mathbf{x})=\left\langle M_{y}(R ; \mathbf{x})\right\rangle_{y \subseteq x, y \text { finite }}
$$

where $M_{y}(R ; x)=\left\{\{e\}^{E(R)}\left(x, y_{1}, \ldots, y_{n}\right) ; e \in w,\left\{y_{1}, \ldots, y_{n}\right\} \subseteq y\right\}$ $\left(\{e\}^{E(R)}\right.$ is tile partial function in $E(R)$-recursion theory with index e. We give $\{e\}^{S(R)}$ the same meaning for $S(R)$-recursion, $\{e\}^{K}$ will mean the Kleene-recursive function with index e).

If we let $I=\operatorname{type}(k), a \in I$ and $F$ a functional of type $k+2$ we prove that

a For $A \subseteq I, A$ is Kleene-recursive in $k+2 F, a, k+2 F$ if and only if $A \in \mathbb{M}_{a}(F ; I)$ 
b For $A \subseteq I, A$ is Kleene-semirecursive in ${ }^{k+2} \mathrm{~F}, a,{ }^{k+2} \mathrm{E}$ if and only if $A$ is $\Sigma_{a}^{*}$-definable over $\operatorname{Spec}(F)$ (if and only if for some $\Delta_{0}$-formula $\varphi$ with parameters from $M_{a}(F ; I)$

$b \in A \Leftrightarrow \exists x \in \mathbb{N}\{a, b\}(F ; I) \varphi(x, b))$.

The superjump ${ }^{k+3} \mathrm{~S}$ is defined as the funcional

$$
k+3_{S(e, F)}=\left\{\begin{array}{llll}
0 & \text { if } & \{e\}(F) & \text { has a value }(\{e\}(F) \downarrow) \\
1 & \text { if }\{e\}(F) & \text { does not have a value }(\{e\}(F) \uparrow)
\end{array}\right.
$$

$k+3_{S}$ is not a normal functional. Recursion in ${ }^{k+3}{ }_{S}$ does not satisfy stage comparison and that a subset of $I$ is recursive in $k+3_{S}$ if and only if both it and it's complement are semirecursive. The reason for this misbehaviour seems to be that ${ }^{k+3} \mathrm{~S}(e, F)$ is defined only for total $F$, while we need information only from a part of $F$ to compute ${ }^{k+3} S(e, F)$. In E-recursion there are two natural candidates for the jump, either a complete $\Sigma^{*}$-definable set, or the spectrum itself. In defining S-recursion we choose the latter.

\section{Definition.}

Define S-recursion from E-recursion by adding the following scheme:

$$
\begin{aligned}
& \{e\}(x, \vec{y})=\operatorname{Spec}\left(\lambda z\left\{e_{1}\right\}(x, \vec{y}, z) ; x\right) \text { if } \lambda z\left\{e_{1}\right\}(x, \vec{y}, z) \text { is total on } \\
& \text { it's spectrum over } x \quad e=\left\langle 8, e_{1}, n\right\rangle
\end{aligned}
$$

As usual, we identify a function with its graph. S-recursion is, like E-recursion, relativezed to arbitrary relations.

Remark. It is essential that we require that $\lambda z\left\{e_{\eta}\right\}(x, \vec{y}, z)$ is total on its spectrum. If we remove that requirement, we may let $e_{1}$ be the index for diagonalization $\left\{e_{1}\right\}\left(e_{2}, a\right)=\left\{e_{2}\right\}(a)$. Then

$$
\operatorname{spec}\left(\lambda\left\langle e_{2}, a\right\rangle\left\{e_{1}\right\}\left(e_{2}, a\right) ; I\right) \text { would have as an element }
$$$$
\left\{\left\langle e_{2}, a\right\rangle:\left\{e_{2}\right\}(a) \simeq 0\right\} \text {. But that set cannot be recursive. }
$$ 
On the other hand, there is no justification for requiring that $\lambda z\left\{e_{1}\right\}(x, \vec{y}, z)$ is defined outside its spectrum.

Inspecting the inductive definition of the $S(R)$-computations we see that

$$
\begin{aligned}
& \left\{\left\langle 8, e_{1}, n\right\rangle\right\}^{S(R)}(x, \vec{y}) \simeq\left\langle M_{y}\right\rangle_{y \in x^{f}}^{f} \text { iff } \\
& \forall z \in \mathbb{M}\left(=\bigcup_{y \in x^{f}} M_{y}\right)\left\{e_{1}\right\}^{S(R)}(x, \vec{y}, z) \downarrow
\end{aligned}
$$

and

$$
\forall y \in x^{f}\left(z \in \mathbb{M}_{y} \Rightarrow \exists e \in w\left(z=\{e\}^{\Phi(F} e_{1}\right)(\vec{y}, x)\right.
$$

and

$$
\text { each } M_{y} \text { is rudimentary closed relative to } F_{e_{1}}
$$

and

$$
\left\langle M_{y}\right\rangle_{y \in x^{f}} \vDash \Sigma^{*}\left(F_{e_{1}}\right) \text {-collection }
$$

where $x^{f}$ means the set of finite subsets of $x, F_{e_{1}}=\lambda z\left\{e_{1}\right\}^{S(R)}\left(x_{3}, y_{y} z\right)$. The length of this computation will then naturally be $\operatorname{Sup}\left\{\alpha,\left\|\left\langle e_{1}, x, \vec{y}, z\right\rangle\right\|^{S(R)} ; \alpha \in \operatorname{Spec}\left(F_{e_{1}} ; x\right)\right.$ and $\left.z \in \operatorname{Spec}\left(F_{e_{1}} ; x\right)\right\}$.

Definition. Let $\mathrm{R}$ be a relation, $\mathrm{x}$ e set, $\mathrm{y} \in \mathrm{x}^{\mathrm{f}}$

$$
\begin{aligned}
& \frac{\operatorname{SM}_{y}(R ; x)}{S-\operatorname{Spec}(R ; x)}=\left\{\{e\}^{S(R)}\left(y_{1}, \ldots, y_{n}, x\right) ;\left\{y_{1}, \ldots, y_{n}\right\} \subseteq y, e \in W\right\} \\
& \left.S M_{y}(R ; x)\right\rangle \\
& y \in x^{f}
\end{aligned}
$$

\section{S-recursion and the Superjump.}

In this section we will let $I=t p(k)$ for some fixed $k \geq 0$. We also let $F$ be a functional of type $k+2$. Before we can prove our main reduction theorem for $S(F)$-recursion, we need some machinery for companion-theory. 
Definition. Let $A \subseteq I \times I$. Assume that $A$ is a transitive relation. Define $\simeq$ by $a \simeq b$ if $A(a, b)$ and $A(b, a)$. We say that $A$ is $a$ code for a set $x$ if $A / \simeq$ is isomorphic to $\langle T C(x), \epsilon\rangle$. Suitable references to the theory of codes will be Sacks [12] and [13], and Normann $[10]$ and $[11]$.

Lemma 1. The relation 'A is a code' is recursive in ${ }^{\mathrm{k}+3} \mathrm{~S}$.

Proof. The relation 'A is a code' may be defined by some quantifiers over $I+$ 'A is a well-founded relation'. Since ${ }^{k+2} E$ is recursive in $k+3_{S}$, the lemma is trivial for $k>0$. For $k=0$ : The relation 'A is well founded' is semirecursive in $2_{E}$, and since $3_{S}$ is a jump-operator it will be recursive in ${ }^{3}$. Lemma 2. There is a function $f$ partially recursive in ${ }^{k+3} \mathrm{~S}, \mathrm{~F}$ such that if $A_{1}, \ldots, A_{n}$ are codes for sets $x_{1}, \ldots, x_{n}$ and $\{e\}^{S(F)}\left(x_{1}, \ldots, x_{n}\right) \downarrow$, then $\lambda a, b f\left(e,\left\langle A_{1}, \ldots, A_{n}\right\rangle, a, b\right)$ is a total characteristic function for a code for $\{e\}^{S(F)}\left(x_{1}, \ldots, x_{n}\right)$. If $A_{1}, \ldots, A_{n}$ are codes for $x_{1}, \ldots, x_{n}$ and $\{e\}^{S(F)}\left(x_{1}, \ldots, x_{n}\right) \uparrow$, then

$$
f\left(e,\left\langle A_{1}, \ldots, A_{n}\right\rangle, a, b\right) \text { will not } b \in \text { defined for any } a, b .
$$

Proof. We use the recursion-theorem, and define $f$ by induction on the length of the computation $\{e\}^{S(F)}\left(x_{1}, \ldots, x_{n}\right)$. There will be 8 cases, according to the type of the index e. The proof is by standard manipulations on codes (see Sacks [13] or Normann [10]) in all cases except scheme 8 :

$$
\{e\}(x, \vec{y})=\operatorname{Spec}\left(\lambda z\left\{e_{1}\right\}(x, \vec{y}, z) ; x\right) .
$$

As an induction hypothesis, assume that $f$ is defined and recursive and acts as it shall for all shorter computations. 
Let codes for $x, \vec{y}$ be given. What we will do will be uniformly recursive in these codes.

$$
\text { Let } F_{e_{\eta}}=\lambda z\left\{e_{\eta}\right\}(x, \vec{y}, z) \text {. }
$$

The idea is to define another function $G$ which is total, recursive in $k+3, S, F$ and the codes and 'equivalent' to $F_{e_{i}}$ Then we can apply ${ }^{k+3_{S}}$ on $G$ to define $\operatorname{Spec}(G)=\operatorname{Spec}\left(F_{e_{1}}\right)$.

Claim 1. Let $A$ be a code for a computation tree $T$ for some $\{e\}^{F(R)}(\vec{y}, x)$-computation for $\vec{y} \in x^{f}$. We may then recursively decide if we may replace $R$ by $F_{e_{1}}$ in the computation or not.

Proof. We here assume that $F_{e_{1}}$ is total on $\operatorname{spec}\left(F_{e_{1}}\right)$. If $F_{e_{1}}$ diverges on some critical argument in $\mathrm{T}$, our procedure will diverge. We will use the recursion theorem to define the following recursive function $\rho$ on $A$.

Let $a \in$ field $A$. We will let $\rho(a)=1$ if we in the computation coded by a have used a part of $R$ different from $F_{e_{1}}$ Otherwise we will let $\rho(a)=0$.

$\rho$ is precisely defnined this way:

If a codes a computation $\sigma$ and for some code $b$ for a subcomputation $T$ of $\sigma, \rho(b)=1$, then $\rho(a)=1$ 。

If for all codes $b$ for subcomputations $\tau$ of $\sigma, \rho(b)=0$, and $\sigma$ is not an application of $R$, let $p(a)=1$. (This takes care of the initial computation.)

If for all codes $b$ for subcomputations $\tau$ of $\sigma, \rho(b)=0$ and $v$ is an application of $R$, we must check if this application actually is an application of $\mathrm{F}_{\mathrm{e}_{1}}$. We may assume as an induction-hypothesis that all applications of $R$ in subcomputations of $\sigma$ actually are applications of $F_{e_{1}}$. Let the application be $Z_{1} \cap R$. We get a 
code for $z_{1}$ and $z_{1} \in \operatorname{Spec}\left(F_{e_{1}}: x\right)$. So $F_{e_{1}}$ is total on $\operatorname{dom}\left(z_{1}\right)=\left\{y_{1} ; \exists y_{2}\left\langle y_{1}, y_{2}\right\rangle \in z_{1}\right\}$. Using $f$, the code for $z_{1}$, and standard manipulations on codes, we may compute a code for $z_{1} \cap F_{e_{1}}$. From $T$ we have a code for $z_{1} \cap \mathrm{R}$. If these two codes code the same set, let $\rho(a)=0$, otherwise, Let $\rho(a)=1$.

Now A codes a computation in $F_{e_{1}}$ if $\rho$ is constant $O$ on $A$. Cleim 1.

Now, define

$$
G(A, B)=\left\{\begin{array}{l}
0 \text { if } A \text { is a code for a computation-tree in } \\
E\left(F_{e_{1}}\right) \text {-recursion leading from } x \text { and some } y \in x^{f} \\
\text { to a set } z, \text { and } B \text { is a code for } F_{e_{1}}(z) . \\
\text { otherwise }
\end{array}\right.
$$

Claim 2. $G$ is recursive in ${ }^{\mathrm{k}+3} \mathrm{~S}, \mathrm{~F}$ and the codes.

Proof. We use the same assumptions as in claim 1. If they do not hold, our procedure for computing $G$ will give a partial functional.

We will describe an algorithm for computing $G$.

Let $A, B$ be given. First decide of both $A$ and $B$ are codes. If they are not, let $G(A, B)=1$. Assume they are codes.

We have already noticed that well-foundedness is recursive in $k+3 S$, so we may recursively decide if $A$ is the code of a relativized computation-tree or not. If not, let $G(A, B)=1$. If it is, we may by claim 1 decide if $A$ is coding a tree for a computation relative to $F_{e_{1}}$. If not, let $G(A, B)=1$. If it is, we get a computation tree $T$ computing a set $z$ in $\operatorname{Spec}\left(\mathrm{F}_{\mathrm{e}_{1}} ; \mathrm{x}\right)$, and we may from $A$ effectively compute a code $C$ for $F_{e_{1}}(z)$. If $B$ and $C$ code the same set, we let $G(A, B)=0$. Otherwise we let $G(A, B)=1$ 。 This ends the proof of claim 2. 
Now, let $A, \vec{B}$ be the codes for $x, \vec{y}$ resp. Let $C$ be a complete r.e. $-G, A, \vec{B}$ subset of $I$ o Using ${ }^{k+3} S$ we see that $C$ will be recursive in ${ }^{k+3} S, F$ uniformly in $A, \vec{B}$. From $C, G$ we may effectively construct a code for $\operatorname{Spec}(G, A, \vec{B} ; I)$.

$x \in \operatorname{Spec}(G, A, \vec{B} ; I)$ since $A$ is a code for $x$ 。

Let $\left\langle M_{a}\right\rangle_{a} \in I=\operatorname{Spec}(G, A, \vec{B} ; I)$. For $z \in \operatorname{Spec}\left(F_{e_{1}} ; x\right)$ the following definitions of the relation ' $F_{e_{1}}(z)=u$ ' are valid

$$
\begin{aligned}
F_{e_{1}}(z)=u^{\prime} \Leftrightarrow & \forall b\left(z, u \in M_{b} \Rightarrow \exists C, B \in M_{b}(B \text { is a code for } u,\right. \\
& C \text { is a code for a computation from } x \text { and some } \\
& \left.y \in x^{f} \text { leading to } z \text {, and } G(C, B)=0\right) \\
\Leftrightarrow & \forall b\left(z, u \in M_{b} \Rightarrow \forall C, B \in M_{b}(B \text { is a code for } u,\right. \\
& C \text { is a code for a computation from } x \text { and some } \\
& \left.y \in x^{f} \text { leading to } z \Rightarrow G(C, B)=0\right)
\end{aligned}
$$

This shows that $\operatorname{Spec}\left(\mathrm{F}_{e_{1}} ; \mathrm{x}\right)$ will be $w-\Sigma^{*}(G)$-definable over $\operatorname{Spec}(G, A, \vec{B} ; I)$. But then we may extract a code for $\operatorname{Spec}\left(F_{e} ; x\right)$ from $A$ and $\operatorname{Spec}(G, A, \vec{B} ; I)$.

By the effectiveness of these arguments we may use the recursion theorem to prove lemma 2 .

Theorem 1. Let $I=t p(k), F$ a functional of type $k+2$ 。

a The relation

$$
\left\{(e, a):\{e\}^{S(F)}(a, I) \downarrow\right\}
$$

is semirecursive in ${ }^{\mathrm{k}+3} \mathrm{~S}, \mathrm{~F}$.

b If a subset $A$ of $I$ is $S(F)$-recursive in $a \in I$, then $A$ is recursive in ${ }^{k+3} \mathrm{~S}, \mathrm{~F}$, a.

c If a subset $A$ of $I$ is $S(F)$-semirecursive in $a \in I$, then $A$ is semirecursive in ${ }^{k}+3 \mathrm{~S}, F, a$. 
Our next result will show that the recursive sets will be the same in the two theories. This will not hold for semirecursion.

Theorem 2. There is a primitive recursive function $\rho$ such that if

$$
\{e\}^{K}\left(F,{ }^{k+3}, \vec{f}, \vec{a}\right) \simeq k
$$

then

$$
\{\rho(e)\}^{S(F)}(\vec{f}, \vec{a}, I) \simeq k
$$

Proof. We will use the recursion theorem for primitive recursion. In all cases except when we apply $k+3 \mathrm{~S}$ we will just imitate what happens in Kleene-recursion. When the Kleene computation seems to apply ${ }^{k+3} S$, we will in the $S(F)$-theory forget the requirements of totality and thereby introxuce some more computations.

So we regard the case

$$
\{e\}\left(F,{ }^{k+3} S, \vec{f}, \vec{a}\right) \simeq{ }^{k+3} S\left(\lambda f\left\{e_{1}\right\}\left(F,{ }^{k+3} S, f, \vec{f}, a\right)\right)
$$

Let $\rho(e)$ be an index for the following $S(F)$-computation:

$$
\text { Find } \operatorname{Spec}\left(\lambda f\left\{\rho\left(e_{\eta}\right)\right\}^{S(F)}(f, \vec{f}, I)\right)
$$

and by inspection compute ${ }^{k+3} S(G)$ for any total extension $G$ of

$$
\lambda f\left\{\rho\left(e_{1}\right)\right\}^{S(F)}(f, \vec{f}, I)
$$

(All computation-trees for $G$ will be in

$$
\left.\operatorname{Spec}(G ; I)=\operatorname{Spec}\left(\lambda f\left\{\rho\left(e_{\uparrow}\right)\right\}^{S(F)}(f, \vec{f}, I)\right)\right)
$$

This ends the proof of theorem 2.

We have now verified that the concepts of total recursion are the same for Kleene-recursion in ${ }^{k+3} \mathrm{~S}$ and S-recursion over type(k)。 our next task is to show that semi-recursion in S-recursion behaves better than semirecursion in ${ }^{k+3} \mathrm{~S}$. This is shown by proving that S-recursion satisfies stage comparison. 
Iemma 3. Iet $R$ be a relation. Uniformly in $R$ there is an index e such that for any pair $\sigma, \tau$ of computation-tuples

$$
\{e\}^{S(R)}(\sigma . \tau) \simeq \begin{cases}0 & \text { if }\|\sigma\|^{S(R)}<\|\tau\|^{S(R)} \text { (where }\|\tau\|^{S(R)}=\infty \text { if } \tau \uparrow ! \\ 1 & \text { if }\|\tau\|^{S(R)} \leq\|\sigma\|^{S(R)} \text { and }\|\tau\|^{S(R)}<\infty .\end{cases}
$$

Proof. We will drop the superscript $S(R)$. We define e by use of the recursion theorem. The definition is by 64 cases according to the schemes used in $\sigma$ and $\tau$. The 49 cases where there is no use of scheme 8 are treated as in E-recursion. (Normann [11]) Moreover, all cases where one of the computations is an initial one, are trivial. We give case 8.8 , which is the most complicated. Let

$$
\begin{aligned}
& \sigma:\left\{e_{1}\right\}(x, \vec{y}) \simeq \operatorname{Spec}\left(\lambda y\left\{e_{2}\right\}(y, x, \vec{y}) ; x\right) \\
& \tau:\left\{a_{1}\right\}(u, \vec{w}) \simeq \operatorname{Spec}\left(\lambda w\left\{d_{2}\right\}(w, u, \vec{v}) ; u\right)
\end{aligned}
$$

We will assume that either $\sigma \downarrow$ or $\tau \downarrow$, and as an induction hypothesis that the lemma is established for any subcomputation of $\sigma$ or $\tau$. It will be clear from the definition that if both $\sigma$ and $\tau$ diverge, then the described computation on $\sigma$ and $\tau$ will diverge. We will use the assumptions to define enother function $G$ which will be total, and such that $\operatorname{Spec}(G ; x \cup u \cup\{e\} \cup\{u\})$ will contain sufficient information to decide if $\|\sigma\|<\|\tau\|$ or $\|\tau\| \leq\|\sigma\|$. $G$ will be defined just on the ordinals, which is no real restriction. We will let $G(\alpha)$ describe what we, with the help of stage comparison so far, can say about the part of the two spectra that is constructed at level $\alpha$.

For the purpose of this definition, let

$$
\langle X, Y, U, Z\rangle=\{O\} \times X \cup\{1\} \times Y \cup\{2\} \times U \cup\{3\} \times Z
$$


Definition of $G$. Each $G(\alpha)$ will be a tuple $\left\langle\mathrm{X}_{1}^{\alpha}, \mathrm{H}_{1}^{\alpha}, \mathrm{X}_{2}^{\alpha}, \mathrm{H}_{2}^{\alpha}\right\rangle$ where $H_{i}^{\alpha}$ is a partial function on $X_{i}^{\alpha}, x_{1}^{\alpha}$ a family of spaces indexed over $x^{f}$ and $x_{2}^{\alpha}$ a family of spaces indexed over $u^{f}$.

If $\lambda$ is a limit ordinal, we let $X_{i}^{\lambda}=\bigcup_{\gamma<\lambda} X_{i}^{\gamma}, H_{i}^{\gamma}=\bigcup_{\gamma<\lambda} H_{i}^{Y}$. (It will follow from the construction that this makes sense.)

To compute $G(\alpha+1)$ we regard two cases

Case 1. If $X_{1}^{\alpha}=\operatorname{Spec}\left(H_{1}^{\alpha} ; x\right)$ or $X_{2}^{\alpha}=\operatorname{Spec}\left(H_{2}^{\alpha} ; u\right)$, let $G(\alpha+1)=G(\alpha)$.

Case 2. Otherwise. We define $x_{1}^{a+1}$ as follows.

For $x_{1} \in x^{f}$, let

$\left(x_{1}^{\alpha+1}\right)_{x_{1}}=\left\{\{s\}^{E\left(H_{1}^{\alpha}\right)}\left(\vec{x}_{1}, x\right) ; s \in w \wedge\left\|\left\langle s, \vec{x}_{1}, x\right\rangle\right\|^{E\left(H_{1}^{\alpha}\right)} \leq \alpha\right\}$

For $u_{1} \in u^{f}$, let

$\left(x_{2}^{\alpha+1}\right)_{u_{1}}=\left\{\{s\}^{E\left(H_{2}^{\alpha}\right)}\left(\vec{u}_{1}, u\right) ; s \in w \wedge\left\|\left\langle s, \vec{u}_{1}, u\right\rangle\right\|^{E\left(H_{2}^{\alpha}\right)} \leq \alpha\right\}$

For $y \in x_{1}^{\alpha+1}$, let

$\mathrm{H}_{1}^{\alpha+1}(\mathrm{y})=\mathrm{z}$ if $\exists \mathrm{w} \in \mathrm{X}_{2}^{\alpha+1}\left(\{e\}\left(\left\langle e_{2}, \mathrm{y}, \mathrm{x}, \overrightarrow{\mathrm{y}}\right\rangle,\left\langle\mathrm{a}_{2}, \mathrm{w}, \mathrm{u}, \overrightarrow{\mathrm{v}}\right\rangle\right)=0\right.$

$\left.\wedge\left\{e_{2}\right\}(y, x, \vec{y})=z\right)$

For $w \in X_{2}^{\alpha+1}$ let

$H_{2}^{\alpha+1}(w)=q$ if $\exists y \in x_{1}^{\alpha+1}\left(\{e\}\left(\left\langle d_{2}, w, u, \vec{v}\right\rangle,\left\langle e_{2}, y, x, \vec{y}\right\rangle\right)=0\right.$

$\left.\wedge\left\{d_{2}\right\}(w, u, \vec{v})=q\right)$

It is E-recursive to decide between case 1 and 2.

Letting $G(0)=\langle\varnothing, \varnothing, \varnothing, \varnothing\rangle$ we use the induction hypothesis and the assumption to prove the following by induction on $\alpha$ :

a $x_{1}^{\alpha}$ is an initial segment of $\operatorname{Spec}\left(\lambda y\left\{e_{2}\right\}(y, x, \vec{y}) ; x\right)$ 
b $\quad X_{2}^{\alpha}$ is an initial segment of $\operatorname{Spec}\left(\lambda w\left\{a_{2}\right\}(w, u, \vec{v}) ; u\right)$

if the latter exists

c For $\mathrm{y} \in \mathrm{x}_{1}^{\alpha}$,

$\lambda w \in X_{2}^{\alpha}\left(\{e\}\left(\left\langle e_{2}, \bar{y}, x, \vec{y}\right\rangle,\left\langle d_{2}, w, u, \vec{v}\right\rangle\right)\right.$ is total, and if the value of the computation is 0 for some $w$ then $\left\{e_{2}\right\}(y, x, \vec{y}) \downarrow$

d For $w \in x_{2}^{\alpha}$

$\lambda y \in X_{1}^{\alpha}\left(\{e\}\left(\left\langle d_{2}, w, u, \vec{v}\right\rangle,\left\langle e_{2}, y, x, \vec{y}\right\rangle\right)\right.$ is total, and if the value of the computation is 0 for some $y$ then $\left\{d_{2}\right\}(w, u, \vec{v}) \downarrow$

e For at least one $i \in\{1.2\}$ is $H_{i}^{\alpha}$ total on $X_{i}^{\alpha}$.

The proof is straightforward.

So $G$ will be recursive in $\tau, \sigma, x, u, \vec{y}, \vec{v}$ and $G$ will be total.

Let $M=\operatorname{Spec}(G ; x \cup u \cup\{x\} \cup\{u\})$.

Claim. At least one spectrum obtained by $\sigma$ or $\tau$ will be included in $M$ 。

Proof. Let $\alpha=$ On $\cap$ M. We regard two cases.

Case 1. In defining $G(\alpha+1)$ we are in case 1.

By symmetry we may assume that $X_{1}^{\alpha}=\operatorname{Spec}\left(H_{1}^{\alpha}\right)$.

Since $G \cap M$ is definable from $G$ and $M$ we see that $H_{1}^{\alpha} \cap \mathbb{M}$ is definable from $G$ and $M$. But then $\operatorname{Spec}\left(H_{1}^{\alpha}\right) \subseteq M$, so $x_{1}^{\alpha} \subseteq M$.

Case 2. In defining $G(\alpha+1)$ we are in case 2 .

By the argument of case 1 , this actually means that both $\mathrm{H}_{1}^{\alpha}$ and $\mathrm{H}_{2}^{\alpha}$ are partial. But this is impossible by $\underline{e}$ above. 
By inspection of $M$ and $M \cap G$ one may decide the proper value of $\{e\}(\sigma, \tau)$ 。

The other cases where scheme 8 is involved, are treated in the same manner, by a recursive function $G$ one tries to imitate the construction of the actual spectrum until either the spectrum is completed or the other computation terminates.

This ends the proof of lemma 3.

As usual we now obtain Gandy's selection theorem for numbers, and that a set is recursive in some parameters if and only if both it and its complement are semirecursive in the parameters. We also have established sufficient properties to claim that $\mathrm{S}^{\mathrm{k}+2}(\mathrm{~F})$-recursion theory over $t p(k)$ is equivalent to Harrington's strong recursion in ${ }^{k+3} \mathrm{~S}, \mathrm{k}+2 \mathrm{~F}$.

\section{Functions and relations}

One of the properties of set-recursion is that for any relation $R$, there is a function $F$ of tjpe $k+2$ such that $E(R)$-recursion over type $k$ is the same as $E(F)$-recursion over type $k$. This is established for $k=0$ in Harrington-Kechris-Simpson [7] and for $k=0$ independently in Kechris [8] and Normann [10]. For a general proof, see Normann [11].

We will prove that this result also is true for S-recursion. We prove the result for $I=t p(k)$, but it may be proved with the same generality as the parallell result in Normann [11].

Definition. Let $R$ be a relation. Define $F_{R}$ by

$$
F_{R}(f)= \begin{cases}0 & \text { if } f \text { is a code for a set } x \text { and } R(x) \\ 1 & \text { otherwise. }\end{cases}
$$


where we say that $f: I \rightarrow w$ is a code if $f$ is the characteristic function of a code.

Iemma 4. $F_{R}$ is $S$-recursive in $I$ relative to $R$. Proof. By lemma 1, the relation ' $f$ is a code' is $k+3$ S-recursive, and thus S-recursive by theorem 2. In E-recursion there is an index $e$ such that if $f$ is a code for $x$, then $\{e\}(f, I)=x$. The lemma then follows.

We cannot prove the other direction of the lemma, since $R$ may contain information about sets not codable. But for our purpose it will be sufficient to do so for $R \cap S-\operatorname{Spec}(R)$.

Lemma 5. In $S(R)$-recursion there is an index $e$ such that if for some $a \in I$ and some $x,\left\{e_{1}\right\}^{S(R)}(a, I)=x$, then $\{e\}^{S(R)}\left(e_{1}, a, I\right)$ is a code for $x$.

Proof. We define $\epsilon$ by the recursion-theorem. We may use the same argument as in lemma 2, except in case 6, relativization to $R$. But there we may use lemma 4.

\section{Theorem 3.}

a $\quad \operatorname{S-Spec}\left(F_{R}\right)=\operatorname{S-Spec}(R)$ for any relation $R$

$\underline{b}$ For $a \in I, A \subseteq I$

$A$ is $S(R)$-recursive in $a, I \Leftrightarrow A$ is $S\left(F_{R}\right)$-recursive in a, I $\left(\Longleftrightarrow A\right.$ is $K$ leene-recursive in $a, F_{R},{ }^{k+3} S$ )

c For a $\in I, A \subseteq I$

$A$ is $S(R)$-semirecursive in $a, I \Leftrightarrow A$ is $S\left(F_{R}\right)$-semirecursive in $a, I$

$\left(\Leftrightarrow A\right.$ is strongly semirecursive in $a, F_{R}, k+3 S$ ) 
Proof.

a Let $x=\left\{e_{1}\right\}^{S\left(F_{R}\right)}(a, I)$. By lemma 4, there is an index e $e_{2}$ such that $x=\left\{e_{2}\right\}^{S(R)}(a, I)$.

Now, let $y=\left\{e_{2}\right\}^{S(R)}(a, I)$. From the prosf of lemma 5 we see that the construction of a code for $y$ is actually a computation in $F_{R}$. But then $y$ is $S\left(F_{R}\right)$-recursive in a, I.

b This is just a special case of $\underline{a}$.

c To obtain $\subseteq$ we need the following claim:

A subset $A$ of $I$ is $S(R)$-semirecursive in $a, I$ if and only if there is a set $Q$ recursive in $a, I$ such that

$b \in A \Longleftrightarrow \exists x \in S M\langle a, b\rangle(R) Q(b, x)$

Proof. If $A=\left\{b ;\{e\}^{S(R)}(a, b, I) \downarrow\right\}$, let

$$
Q(b, x) \Longleftrightarrow x \text { is a computation-tree for }\{e\}^{S(R)}(b, a, I) \text {. }
$$

On the other hand, let $Q$ be given and let $A$ be defined from $Q$ as above. By the Gandy selection operator obtained from lemma 3, we describe a partial function recursive in $a, I$ and defined just on $A$.

5. Equivalences to the $S(R)$-theories

The following considerations are valid for most notions of computation-theories, see e.g. Fenstad [1] or [2] or Moldestad [9]. So, let $\oplus$ be a computation-theory on a computation domain $I$. We say that $\oplus$ is $p$-normal if we ${ }^{\oplus}$-recursively may compare lengths of computations in $\Theta$, i.e. $\Theta$ satisfies lemma 3 of this paper. If $\otimes$ is p-normal, $\Theta$ will allow a selection operator for numbers (Grilliot [4], see also Moldestad [9]). 
Definition. Let $\Theta$ be a computation-theory on the computationdomain $I$. We call $\oplus$ weakly normal if $=$ on $I$ is $\Theta-r e c u r s i v e$, $\Theta$ is p-normal and there is an index e such that

$$
\{e\}_{\Theta}\left(e_{1}, \vec{a}\right)=\left\{\begin{array}{lll}
0 & \text { if } & \forall b \in I \quad\left\{e_{1}\right\}_{\Theta}(\vec{a}, b)=0 \\
1 & \text { if } \forall b \in I \quad\left\{e_{1}\right\}_{\Theta}(\vec{a}, b) \downarrow \text { and } \exists b \in I\left\{e_{1}\right\}_{\Theta}(\vec{a}, b) \neq 0 .
\end{array}\right.
$$

We define the notion of a code over I as in section 3, and we define $\underline{\operatorname{Spec}(\Theta)}=\left\langle\mathbb{M}_{a}(\Theta)\right\rangle_{a \in I}$ by

$x \in \mathbb{M}_{a}(\Theta) \Leftrightarrow$ there is a code for $x$ that is $\Theta[a]$-recursive.

Let $R_{\circledast}=\left\{\langle\sigma, \alpha\rangle ; \sigma \in \Theta \wedge\|\sigma\|_{\Theta}=\alpha\right\}$.

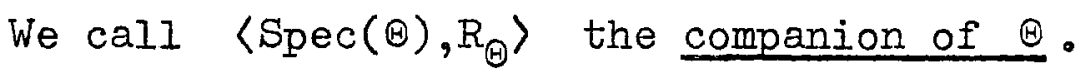

Remark. Spec(ब) will be $E\left(R_{\Theta}\right)$-recursively closed and satisfies $\Sigma^{*}\left(R_{\Theta}\right)$-collection.

Lemma 6. A subset $A$ of $I$ is $\Theta[a]-$ semi-computable if and only if it is $\Sigma^{*}\left(R_{\Theta}, a\right)$ definable over $\operatorname{Spec}(\Theta)$.

Proof. Assume A is $\Theta[a]$-semicomputable,

$$
b \in A \Longleftrightarrow\{e\}_{\Theta}(a, b) \downarrow \text { for some a. }
$$

Then

$$
\mathrm{b} \in \mathrm{A} \Leftrightarrow \exists n \in \mathbb{N} \quad \exists a \in \mathbb{M}_{a, b}(\Theta)\left(\langle\langle e, a, b, n\rangle, \alpha\rangle \in R_{\Theta}\right)
$$

On the other hand, let $A$ be $\Sigma^{*}\left(R_{\Theta}, a\right)$-definable. Let $\varphi$ be a $\Delta_{0}$-formula such that

$$
\begin{aligned}
& b \in A \exists x \in \mathbb{M}_{a, b}(\Theta) \varphi\left(x, a, b, R_{\Theta}\right) \\
& \Leftrightarrow \Leftrightarrow e \in \mathbb{N}(e \text { is an index for a code for a set } x \\
& \text { such that } \varphi\left(x, a, b, R_{\Theta}\right) .
\end{aligned}
$$

Given a code for $x$, we may decide $\varphi\left(x, a, b, R_{\Theta}\right) \quad E\left(R_{\circledast}\right)$-recursively 
in that code. The relation ' $A$ is a code' is semirecursive in E-recursion, and we may compute $x$ uniformly in a code for $x$. By Gandy-selection we see that $A$ is semirecursive.

\section{Definition.}

a Let $a$ be an ordinal. Let $f: c \rightarrow a$. We call $f$ normal if $f$ is strictly monotone and continuous.

b Let $\left\langle M_{a}\right\rangle_{a \in I}$ be a family of structures indexed over $I, R$ a relation. $\left\langle M_{a}\right\rangle_{a \in I}$ is R-admissible over $I$ if aach $M_{a}$ is rudimentary closed in $R$, and $\left\langle\mathbb{M}_{a}\right\rangle_{a \in I}$ satisfies $\Sigma^{*}$-collection over I. A function $f: M \rightarrow M$ is closed in $\left\langle\mathbb{M}_{a}\right\rangle_{a \in I}$ if for each $a \in I$, if $x \in \mathbb{M}_{a}$ then $f(x) \in \mathbb{M}_{a}$. $f$ is $w-\Delta^{*}$ if the graph of $f$ is weakly $\Delta^{*}$-definable. c Let $\left\langle M_{a}\right\rangle_{a \in I}$ be a family R-admissible over $I$. $\left\langle M_{a}\right\rangle_{a \in I}$ is weakl R-Mahlo if the following is satisfied: Let $\alpha=\sup \left(\right.$ On $\left.\cap M_{a} ; a \in I\right)$

Let $f: \alpha \rightarrow \alpha$ be normal, closed in $\left\langle M_{a}\right\rangle_{a \in I}$ and weakly $\Delta^{*}(R)$. Then there is a family $\left\langle\mathbb{N}_{a}\right\rangle_{a \in I}$ R-admissible over $I$ such that $f$ is closed in $\left\langle\mathbb{N}_{a}\right\rangle_{a \in I}$, for each a $\mathbb{N}_{a} \subseteq \mathbb{M}_{a}$, and for at least one $a \in I$, the inclusion is proper。

(We write this $\left\langle\mathbb{N}_{a}\right\rangle_{a \in I} \varsubsetneqq\left\langle M_{a}\right\rangle_{a \in I}$ )

The following theorem is proved with various degrees of generality in Harrington-Kechris-Simpson [7], Normann [10], Kechris [8] and Moldestad [9].

Theorem 4. Let $\oplus$ be a weakly normal computation-theory on $I$. Then $\Theta$ is equivalent to $E(R)$-recursion in $I$ for some $R$ if and only if $\operatorname{Spec}(\Theta)$ is not weakly $R_{\Theta}$-Mahlo. 
We will prove a similar result for S-recursion.

Definition. Iet $\left\langle\mathbb{M}_{a}\right\rangle_{a \in I}$ be R-admissible over $I$.

a $\left\langle M_{a}\right\rangle_{a \in I}$ is strongly $R$-Mahlo if the following is satisfied: Let $f: a \rightarrow a$ be normal, closed in $\left\langle\mathbb{M}_{\langle a, b}\right\rangle_{b \in I}, f$ is $w-\Delta_{a}^{*}(R)$. Then there is a family $\left\langle N_{b}\right\rangle_{b} \in I$ that is R,a-admissible over I such that $f$ is closed in $\left\langle N_{b}\right\rangle_{b} \in I$ and $\left\langle N_{b}\right\rangle_{b} \in I \in M_{a}$.

b $\left\langle M_{a}\right\rangle_{a \in I}$ is weakly hyper-R-Mahlo if the following is satisfied: Let $f: \alpha \rightarrow \alpha$ be normal, closed in $\left\langle\mathbb{M}_{a}\right\rangle_{a \in I}$ and $w-\Delta^{*}(R)$. Then there is a strongly R-Mahlo iamily $\left\langle N_{a}\right\rangle_{a \in I} \subsetneq\left\langle M_{a}\right\rangle_{a} \in I$ such that $f$ is closed in $\left\langle N_{a}\right\rangle_{a} \in I^{\circ}$

Remark. If $k=0$, then $I=w$ so $\mathbb{M}_{a}=\mathbb{M}_{b}$ for $a I l a, b \in I$. Then these notions coincide with admissible, recursivaly Mahlo and recursively hyper Mahlo. There will be no distinction between the weak and strong Mahlo-property.

Lemma 2. Let $R$ be an arbitrary relation. $S-\operatorname{Spec}(R ; I)$ is the least strongly R-Mahlo family over I.

\section{Proof.}

i $S-\operatorname{Spec}(R ; I)$ is strongly R-Mahlo. Let $S-\operatorname{Spec}(R ; I)=\left\langle M_{a}\right\rangle_{a} \in I^{*}$

Proof. Iet $f$ be $\Delta_{a}^{*}(R)$, normal and closed in $\langle M\langle a, b\rangle\rangle_{b} \in I$. By the Gandy selection operator for numbers, $f$ is $S(R)$-recursive and there is an $S(R)$-recursive function $f_{1}:$ on $\rightarrow$ Codes such that $f_{1}(\alpha)$ is a code for $f(\alpha)$.

Let $G(a, b)=\left\{\begin{array}{lll}1 & \text { if } b \in f_{1}(\alpha) \\ 0 & \text { if } b \notin f_{1}(\alpha)\end{array}\right.$

Then $\operatorname{Spec}(R, G, a ; I) \in M_{2} \cdot \operatorname{Spec}(R, G, a ; I)$ will have the wanted properties. 
ii $S(R)$-recursion is closed within any strongly R-Mahlo family locally of type $k+1$ (or type I) (i.e. $x \in M_{a} \Leftrightarrow x$ has a code in $M_{a}$ )。

Proof. Let $\left\langle M_{a}\right\rangle_{a} \in I$ be strongly R-Mahlo. By induction on the length of computations we will prove that the partial function $\lambda e, \vec{x}\{e\}^{S(R)}(\vec{x})$ is closed in $\left\langle M_{a}\right\rangle_{a \in I}$ and that the relation $\{e\}^{S(R)}(\vec{x}) \simeq y$ is $w-\Sigma^{*}$-definable in $\left\langle M_{a}\right\rangle_{a \in I}$, by proving that the computation-tree also will be in $\mathrm{M}_{\mathrm{a}}$ when $\overrightarrow{\mathrm{x}} \in \mathrm{M}_{\mathrm{a}}$. For all schemes except scheme 8 this is known from E-recurision. So assume $\{e\}^{S(R)}(\vec{x})=\operatorname{Spec}\left(\lambda y\left\{e_{1}\right\}(y, \vec{x}) ; x_{1}\right)$. By the induction hypothesis, the function $\lambda y\left\{e_{1}\right\}(y, \vec{x})$ will be $W-\Sigma^{*}$-definable. in the parameters. Define $f$ by

$$
f(0)=1 \quad f(\lambda)=\bigcup_{\gamma<\lambda} f(\gamma) \text { when } \lambda \text { is a limit. }
$$

Let $f(\alpha+1)$ be the least ordinal $\gamma \geq f(\alpha)+1$ such that we in order to define $\operatorname{Spec}_{f(\alpha)}\left(\lambda y\left\{e_{1}\right\}(y, \vec{x}) ; x_{1}\right)$ only need computations

$$
\left\{e_{1}\right\}(y, \vec{x}) \text { of length }<\psi \text {. }
$$

We use the fact that $\left\langle\mathbb{M}_{a}\right\rangle_{a} \in I$ is locally of type $k+1$ to prove that whenever the parameters are in $M_{a}$, then $f$ is closed in $\left\langle\mathbb{M}_{\langle a, b}\right\rangle_{b \in I}$ and $f$ is $w-\Delta_{a}^{*}$-definable. Let $\left\langle N_{b}\right\rangle_{b \in I} \in \mathbb{M}_{a}$ be R-admissible such that $f$ is closed in $\left\langle N_{b}\right\rangle_{b} \in I$. Then $\lambda y\left\{e_{1}\right\}(\vec{x}, y)$ is $w-\Delta^{*}$-definable over $\left\langle\mathbb{N}_{b}\right\rangle_{b \in I}$, so $\operatorname{Spec}\left(\lambda y\left\{e_{1}\right\}(\vec{x}, y) ; x_{1}\right)$ is a definable subfamily of $\left\langle N_{b}\right\rangle_{b \in I}$ and thus an element of $\mathbb{M}_{a}$. By a similar argument we see that the computationtree will be in $M_{a}$.

In theorem 4 we used the notion of equivalent theories. We must make this notion precise. 
Definition. Let $\Theta_{1}$ and $\Theta_{2}$ be two weakly normal theories on I ( $E$ and $S$-recursion restricted to $I$ may be regarded as such theories) $\Theta_{1}$ and $\Theta_{2}$ are equivalent if $\operatorname{Spec}\left(\Theta_{1}\right)=\operatorname{Spec}\left(\Theta_{2}\right)$ and $R_{\Theta_{1}}$ and $R_{\Theta_{2}}$ are $\Delta^{*}$-definable in each other. The two theories will be equivalent iff the semirecursive sets are the same.

Theorem 5. Let $\Theta$ be a weakly normal theory on I. Then the following two statements are equivalent:

i There is a relation $R$ such that $\otimes$ is equivalent to $S(R)$ recursion over I.

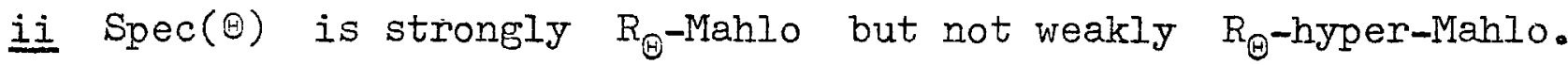

Corollary 5.1. Let $I=\operatorname{tp}(k)$.

Let $\Theta$ be a weakly normal theory on $I$.

Then the two statements are equivalent:

i There is a type $k+2$-functional $F$ such that $\Theta$ is equivalent to stiong recursion in $k+3 \mathrm{~S}, \mathrm{~F}$.

ii Spec( $\Theta)$ is strongly $R_{\Theta}$-Mahlo but not weakly hyper- $R_{\Theta}-$ Mahlo. This is a consequence of theorems 3 and 5.

When $k=0$, we are regarding normal theories over $\mathbb{I N}$, and we then observe:

Corollary 5.2. Let $\Theta$ be a normal theory on $w$. Then $\Theta$ is equivalent to strong recursion in ${ }^{2} F,{ }^{3} S$ for some $F$ if and only if the companion of $\Theta$ is recursively Mahlo, but not recursively hyperMahlo. 


\section{Proof of theorem 5.}

$\underline{i} \Rightarrow \underline{i}$ Let $\Theta$ and $R$ be given, $\operatorname{Spec}(\Theta)=\operatorname{S-Spec}(R ; I)=\left\langle\mathbb{M}_{a}\right\rangle_{a} \in I$, $R_{\oplus}$ and $R$ are $\Delta^{*}$ in each other.

$\Theta$ and $R$ will induce two different hierarchies on $\left\langle M_{a}\right\rangle_{a} \in I$, call them $\left\langle\mathbb{M}_{a}^{\alpha}\right\rangle_{a \in I}$ from $(0)$ and $\left\langle N_{a}^{\alpha}\right\rangle_{a} \in I$ from $S(R)$. Let $R$ be defined from $R_{\Theta}$ by 'the $\Delta^{*}$-formula $\Phi^{\prime}$ and $R_{\Theta}$ from $R$ by 'the $\Delta^{*}$-formula $\psi^{\prime}$ '.

Define $g_{1}(\alpha)=\mu \beta \geq \alpha$ such that $R \cap\left\langle\mathbb{M}_{a}^{\alpha}\right\rangle_{a \in I}$ is definable over $\left\langle M_{a}^{\beta}\right\rangle_{a \in I}$ by $\Phi$.

Define $g_{2}(\alpha)=\mu \beta \geq \alpha$ such that $R_{\Theta} \cap\left\langle N_{a}^{\alpha}\right\rangle_{a \in I}$ is lefinable over $\left\langle\mathrm{N}_{a}^{\beta}\right\rangle_{a \in I}$ by $\Psi$.

$g_{1}$ and $g_{2}$ will be $\Delta^{*}$-definable and closed in $\left\langle M_{a}\right\rangle_{a \in I}$. Moreover, at fix-points $\lambda$ for $g_{1}$ resp. $g_{2}, R \cap\left\langle M_{a}^{\lambda}\right\rangle$ will be $\Delta^{*}\left(R_{\Theta}\right)$-definable over $\left\langle M_{a}^{\lambda}\right\rangle_{a \in I}$ (resp. statement for $R_{\Theta},\left\langle N_{a}^{\lambda}\right\rangle_{a} \in I$ and $R$ ).

We may now show that $\left\langle M_{a}\right\rangle_{a} \in I$ have the same Mahlo-properties w.r.t. $R$ and $R_{\Theta}$.

Let $f$ be a normal $\Delta^{*}$-function. Let $f_{1}=f \div g_{1} \cdot g_{2}$.

$f_{1}$ is normal, $\Delta^{*}$-definable and if $f$ is closed in $\left\langle M_{a}\right\rangle_{a} \in I$, $f_{1}$ is closed in $\left\langle M_{a}\right\rangle_{a \in I}$.

Moreover, a fix-point for $f_{1}$ will be a fix-point for $f, g_{1}$ and $g_{2}$. Claim 1. $\operatorname{Spec}(\Theta)$ is strongly $R_{\Theta}$-Mahlo.

Proof. Let $f$ be a normal $\Delta_{a}^{*}$-function closed in $\left\langle M_{a, b}\right\rangle_{b} \in I$. Since $\left\langle\mathbb{M}_{a}\right\rangle_{a \in I}$ is strongly R-Mahlo (lemma 7), $f_{1}$ is closed in an R-admissible subfamily $\left\langle\mathbb{N}_{\langle a, b}^{\alpha}\right\rangle_{b \in I} \in M_{a}$. But $a$ is a fix-point of $f_{1}$, so $R_{\Theta}$ is $\Delta^{*}$ over $\left\langle N^{\alpha}\langle a, b\rangle\right\rangle_{b \in I}$, so $\left\langle\mathbb{N}_{\langle a, b\rangle}^{\alpha}\right\rangle$ is $R_{\Theta^{-}}$ admissible. 
Claim 2. $\operatorname{Spec}(\Theta)$ is not weakly hyper- $\mathrm{R}_{\Theta}-$ Mahlo.

Proof. Assume it is. By the argument of claim 1 it follows that S-Spec(R,I) is weakly hyper-R-Mahlo, which is false by lemma 7. $\underline{i i} \Rightarrow \underline{i}$ Assume that $\operatorname{Spec}(\Theta)$ is strongly $R_{\Theta}-$ Mahlo but not

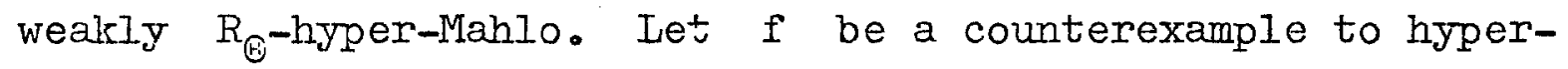
Mahloness. Define

$$
\begin{aligned}
& \mathrm{R}_{\gamma}=\{\langle a, b, \gamma\rangle ; \mathrm{a} \text { and } \mathrm{b} \text { are } \Theta \text {-computations and } \\
& \|\mathrm{a}\|_{\Theta}\left\langle\|\mathrm{b}\|_{\Theta}\langle f(\gamma)\} \text {. Let } \mathrm{R}=\underset{\gamma \in \mathrm{On}_{\gamma}}{\mathrm{R}} \cdot \mathrm{R}\right. \text { is clearly } \\
& \Delta^{*}\left(\mathrm{R}_{\Theta}\right) \text { over } \operatorname{Spec}(\Theta) .
\end{aligned}
$$

Claim 3. Spec(®) is the least strongly R-Mahio family over I.

Proof. Since $\operatorname{Spec}(\Theta)$ is strongly $R_{\Theta^{-M a h l o}}$ and $R$ is $\Delta^{*}\left(R_{\Theta}\right)$ we may use the arguments from $\underline{i} \Rightarrow \underline{i}$ to see that $\operatorname{Spec}(\Theta)$ is strongly R-Mahlo. On the other hand, if there is a subfamily $\left\langle N_{a}\right\rangle_{a \in I}$ that is strongly R-Mahlo, it is sufficient to prove that $f$ is closed in $\left\langle N_{a}\right\rangle_{a \in I}$ and that $R_{\Theta}$ is $\Delta^{*}(R)$-definable over $\left\langle\mathrm{N}_{\mathrm{a}}\right\rangle_{a \in I} \cdot$

a $f$ is closed in $\left\langle\mathbb{N}_{a}\right\rangle_{a \in I}$.

Let $\alpha \in \mathbb{N}_{a}$. Then $\{\langle a, b\rangle: a$ and $b$ are $\Theta$-computations and $\left.\|a\|_{\Theta}<\|b\|_{\Theta}<f(\alpha)\right\} \in \mathbb{N}_{a}$ since $N_{a}$ is rudimentary closed in $R$. But this is a prewellordering of length $f(\alpha)$, so $f(\alpha) \in \mathbb{N}_{a}$.

b $R_{\Theta}$ is $\Delta^{*}(R)$-definable over $\left\langle N_{a}\right\rangle_{a \in I}$ : $\langle a, \alpha\rangle \in R_{\Theta}$ if for some $b \in I,\langle a, b, \alpha+1\rangle \in R$ and $a$ has rank $\alpha$ in the prewellordering $\{\langle c, d\rangle ;\langle c, d, \alpha+1\rangle \in R\}$. This proves claim 3.

By lemma $7, \underline{i \underline{i}} \Rightarrow \underline{i}$ will hold, and theorem 5 is established. 


\section{The sections of $S(R)$-theories}

In Sacks [12] and [13] the notion of abstract $k+1$-sections is defined and it is proved that they are exactly the $k+1$-sections of normal functionals $k+2 F$.

In this section we will give a similar characterization of the $k+1$-section of ${ }^{k+3} \mathrm{~S},{ }^{k+2} \mathrm{~F}$, i.e. the subsets of $t p(k)$ recursive in ${ }^{k}+3_{S}, k+2 F$. Both characterizations and proofs are suitable adjustments of the arguments of Sacks.

In this section we will restrict ourselves to recursion in $I=t p(k)$. The $\underline{b}$-part may however always be generalized to normal recursion on two domains (Moldestad [9]).

\section{Definition of Abstract $k+3$ S-section}

a $\underline{k=0} . A$ is an abstract ${ }^{3}$ S-section if

i A is an abstract 1-section (Sacks [12]) i.e.

Each element in $A$ has a code in $A$

A is admissible and satisfies $\Delta_{0}-D C$

ii If $\varphi$ is a $\Delta_{0}$-formula and $\vec{y} \in A^{n}$ and

$A \vDash \forall x \quad \exists y(x, y, \vec{y})$

then there is an abstract 1-section B such that

$\overrightarrow{\mathrm{y}} \in \mathrm{B} \in \mathrm{A}$ and $\mathrm{B}=\forall \mathrm{x} \exists \mathrm{y} \varphi(\mathrm{x}, \mathrm{y}, \overrightarrow{\mathrm{y}})$

b $\underline{k>0} . A$ is an abstract ${ }^{k+3}$ S-section if there exists a set $B$ such that $A \in B, A$ is countable in $B, A<\Sigma_{1} B$ and both $A$ and $B$ have the following properties:

i. They are rudimentary closed and satisfies $\Sigma_{1}$-collection (admissible with gaps) and are locally of type $k+1$.

ii They are closed under S-recursion. 
Remark. $\underline{i}$ and $\underline{i i}$ play the same rôle in both definitions. gives the appropriate variant of the Mahlo-property.

Theorem 6. Let $F$ be of type $k+2$. Let $x \in A \Leftrightarrow x$ has a code recursive in $F,{ }^{k+3} S$. Then $A$ is an abstract $k+1$-section.

Proof. Let $\left\langle M_{a}\right\rangle_{a \in I}=S-\operatorname{Spec}(F) . A=M_{a}$ for a recursive.

a. $\underline{k}=0$. Here $A=I^{F}$, where $\rho^{F}$ is the least ordinal recursively Mahlo in $F$ (Harrington [5]). Assume

$$
A \vDash \forall x \exists y \varphi(x, y, \vec{y}) \text {. }
$$

Define

$$
g(\gamma)=\mu \beta \forall x \in I_{\gamma}^{F} \exists y \in I_{\beta}^{F} \varphi(x, y, \vec{x}) .
$$

$g$ is closed in some F-admissible ordinal $a_{0}$, and $I_{\alpha_{0}}^{F}$ will be an abstract 1-section.

b $\underline{k}>0$. Let $c$ be a complete $S(R)$-semirecursive subset of $\omega$. Let $B=M_{C} \cdot \quad B y$ Gandy's selection operator for numbers we may use a proof due to Harrington [6] (see also Moldestad [9]) to see that $A<\Sigma_{1} B$. Since $C$ in a way acts as an enumeration of $A$, $A \in B$ and $A$ is countable in $B$. Clearly both $A$ and $B$ are closed under S-recursion.

This proves theorem 6 .

Theorem 7. If $A$ is an abstract ${ }^{k+3} \mathrm{~S}$-section, then there is some functional $F$ of type $k+2$ such that $A$ is the $k+1$-section of $\mathrm{F}, \mathrm{k}+3_{\mathrm{S}}$.

Proof. By theorem 3 it is sufficient to find a relation $P$ such that $A$ is the sets $S(P)$-recursive in $t p(k)$.

a $\underline{k=0}$. We want to find $\mathrm{P}$ such that $\mathrm{A}$ is the least P-recursively Mahlo structure. 
Define a set of conditions $\mathbb{P}$ by

$p \in \mathbb{P}$ if $p \subseteq O n, p \in A$ and no ordinal $\leq \operatorname{rank}(p)$ is $p$-recursively Mahlo. $p \leq q$ if $p=q \cap \operatorname{mn}(p)$.

If $\varphi$ is a $\Delta_{0}$-formula with parameters $\vec{x}$, and $p \in \mathbb{P}$, we say $p$ it $\varphi(\vec{x}, P)$ if $I_{\operatorname{m}(p)}^{p}=\varphi(\vec{x}, p)$.

For other formulas

$$
\begin{aligned}
& p \text { it } \exists x \varphi \text { if for some } x \in L_{m n(p)}^{p} \text { pit } \varphi(x) \\
& p \mathfrak{H} \varphi \vee \psi \text { if } p \mathbb{H} \varphi \text { or } p \mathbb{t} \psi \\
& p \Vdash \neg \varphi \text { if } \forall q \geq p \quad q \not \nvdash \varphi \quad(q \text { does not force } \varphi \text { ) }
\end{aligned}
$$

Let $\mathrm{P}$ be $\mathbb{P}$-generic over $A$ (or actually the union of a $\mathbb{P}$ generic set from $\mathbb{P}$ ).

$\stackrel{i}{ } \quad I_{\operatorname{In}(P)}^{\mathrm{P}}=\mathrm{A}$.

Proof. $I_{r n(P)}^{P} \subseteq A$ since each $p \in A$.

Let $x \in A$, and let $y \subseteq w$ be a code for $x$.

Let $p$ be any condition. Let $q=p \cup\{r m(p)+n ; n \in y\}$

Then $y \in I_{r n(q)+1}^{q}$. Since $P$ is generic, $y$ will be in $I_{m(p)}^{p}$

for some initial $p \subseteq P$, and since $m(P)$ is admissible, $y \in I_{\operatorname{In}(P)}^{P}$.

ii $\operatorname{In}(P)$ is P-recursively Mahlo.

Proof. Assume $\langle A, P\rangle \mid \forall x \exists y \varphi(x, y, P)$.

Since $P$ is generic, there is an initial $p \subseteq P$ such that $\mathrm{p} \Vdash \forall \mathrm{x} \exists \mathrm{y} \varphi(\mathrm{x}, \mathrm{y}, \mathrm{P})$,

so $\forall q \geq p \forall x \quad \exists r \geq q r i r \exists y \varphi(x, y, P)$.

Let $B \in A$ be an abstract 1-section such that $p \in B$ and $\mathrm{B} F \forall \mathrm{q} \geq \mathrm{p} \quad \forall \mathrm{x} \quad \exists \mathrm{r} \geq \mathrm{q} r \mathbb{1} \exists \mathrm{y} \varphi(\mathrm{x}, \mathrm{y}, \mathrm{P})$ 。

Let $p^{\prime}$ be an extention of $p$ that is generic over $B$. 
Then $B=I_{r n}^{p^{\prime}}\left(p^{\prime}\right) \cdot$ Let $\beta \geq m(p)$ be the least admissible ordinal such that

$$
I_{\beta}^{p^{\prime}} \vDash \forall x \exists y \varphi\left(x, y, p^{\prime}\right) \text {. }
$$

Let $q=p^{\prime} \cap \beta$. Then $q \in \mathbb{P}, q \geq p$ and for any proper extention $r>q$,

$$
r \leqslant \exists \text { ( } B \text { is admissible in } P \text { and } \forall x \in B \quad \exists y \leqslant B \varphi(x, y, P) \text { 。 }
$$

Since $P$ is generic, this shows a variant of recursive Mahloness.

b $\underline{k}>0$. Define a set of conditions $\mathbb{P}_{A}\left(\mathbb{P}_{B}\right)$ by

$p \in \mathbb{P}_{A}$ if $p \in A, p \subseteq$ on $\times I$ and for $\alpha=r n(p)$,

$\alpha$ is $S(p)$-recursive in I (without parameters outside $\omega$ )

$q \geq p$ if $q$ is an end-extention of $p$ (i.e. $\langle\beta, a\rangle \in q \backslash p \Rightarrow$ $\beta \geq \operatorname{mon}(p)$.

Let $P$ be $\mathbb{P}_{A}$-generic over $A$. (It will be clear from the argument what sort of generity we need.) We may assume that $P \in B$ since $A$ is countable in $B$.

Let $\left\langle\mathbb{N}_{a}\right\rangle_{a \in I}=S-\operatorname{Spec}(P, I)$, o recursive. We will prove that $N_{0}=A$.

i $A \subseteq N_{0}$.

Proof. Let $x \in A, \quad p$ a condition. Let $y$ be a code for $x$. Let $q=p \cup\{(r n(p), a) ; a \in y\}$.

Clearly $q$ is a condition, and if $P$ extends $q, m(p) \in N_{0}$ (since $\mathrm{p}$ is a condition) and $\mathrm{y} \in \mathrm{N}_{\mathrm{o}} \cdot$ Then $\mathrm{x} \in \mathbb{N}_{0}$ as well. Since $P$ is generic, $x \in N_{0}$.

ii $N_{0} \subseteq A$.

Let $x \in N_{0}$. Then $x=\{e\}^{S(P)}(I)$ for some $e \in \omega$. If this computation is

$$
x=\{e\}^{S(p)}(I)
$$


for some initial condition $p \subseteq P$, then $x \in A$ since $A$ is closed under S-recursion.

If we need cofinally much information about $P$ to compute $x$, the computation still takes place in $B$ since $P \in B$ and $B$ is closed under S-recursion. Let $\alpha$ be the supremum of the ordinals occuring in the computation, and let $P_{0}=P U\langle\alpha, 0\rangle$. Then $P_{0} \in \mathbb{P}_{B}$.

Let $\mathrm{p} \subseteq \mathrm{P}$ be initial. Then

$$
B \vDash \exists q \geq p \quad\{e\}^{S(q)}(I) \downarrow \text {. }
$$

Since $A<\Sigma_{1} B$,

$$
A \vDash \exists q \geq p \quad\{\}^{S(q)}(I) \downarrow \text {. }
$$

This contradicts the fact that $P$ is generic. So $N_{0} \subseteq A$. This ends the proof of theorem 7.

Corollary 7.1. Let $\Theta$ be a weakly normal computation-theory on I such that $\operatorname{Spec}(\Theta)$ is strongly $R_{\Theta^{-M a h l o . ~ T h e n ~ f o r ~ s o m e ~ f u n c t i o n a l ~}}$ $F$ of type $k+2, k+1-s c(\Theta)=k+1-s c(k+3 S, F)$.

Corollary 7.2. For each $k$, there is a normal functional $F$ of type $k+2$ such that

$$
k+1-s c\left({ }^{k+3} S, F\right)=k+1-s c\left({ }^{k+3} E\right) .
$$




\section{References}

1. J.E. Fenstad, Computation theories: An axiomatic approach to recursion on general structures, in: Lecture Notes in Mathematics no 499 (Springer-Verlag 1975) 143-169.

2. J.E. Fenstad, Monograph on generalized recursion theory, to appear.

3. R.O. Gandy, General recursive functionals of finite type and hierarchies of functions, Ann. Fac. Sci. Univ. Clermond-Ferrand No 35 (1967) 5-24.

4. T.J. Grilliot, Selection functions for recursive functionals, Notre Dame Jour. Formal Log.X (1969) 225-234.

5. L.A. Harrington, The Superjump and the first recursively Mahlo ordinal, in Fenstad-Hinman (eds) Generalized Recursion Theory (North Holland, Amsterdam 1974) 43-52。

6. L.A. Harrington, Coritributions to recursion theory on higher types. Ph.D. Thesis, M.I.T. (1973)。

7. I.A. Harrington, A.S. Kechris, S.G. Simpson, 1-envelopes of type 2 objects, American Math. Soc. Notices 20 (1973) A-587.

8. A.S. Kechris, The structure of envelopes, A survey of recursion in higher types, M.I.T. Logic Seminar Notes, December 1973.

9. J. Moldestad, Computations in higher types, Lecture Notes in Mathematics 574, Springer-Verlag 1977.

10. D. Normann, Imbedding of higher type theories, Preprint series in mathematics, No $16-1974$, Oslo.

11. D. Normann, Set recursion, Proceedings from conference in generalized recursion theory, Oslo - 77 .

12. G.E. Sacks, The 1-section of a type $n$ object, in: FenstadHinman (eds) generalized Recursion Theory (North-Holland, Amsterdam 1974) 81-93.

13. G.E. Sacks, The k-section of a type $-n$ object, to appear. 